\title{
Potential health impact of transport traffic restriction in the center of Baku, the capital of Azerbaijan
}

Samir Mehtiyev

${ }^{1}$ Public Health and Reform Center

\begin{abstract}
The transport traffic is known to be the major reason for air pollution in urban areas. Two pollutants should be considered with attention: the particles with diameter of less than 10 microns (PM10) and carbon monoxide. The paper discusses application of time-series and geographical studies to the investigation of air pollution health effects as well as attempts to estimate potential health impact of restriction traffic in the center of Baku by 25\% based on local data published in 2012(4) and 2013(5).

Time-series studies investigate association between short-term variations in air pollution levels and health events counts. Confounding factors that change slowly over the time do not introduce much distortion for the association in question as population is used as its own control.

Geographical studies aimed at investigation of association between long-term exposure to air pollution and chronic health outcomes. They are known to be prone to confounding because they compare populations from different locations. The evidence of air pollution effects from time-series and geographical studies is complementary. The problem of traffic air pollution is being intensified with each year and becomes one of the main public health priorities in Baku. Assuming that the results of six cities study (3) can be generalized to Baku the total number of preventable annual deaths should be around 419 in case of restriction traffic in the center of Baku by $25 \%$. For the low border of $95 \% \mathrm{CI}$ the result is as much as 153 .

Despite uncertainties in assumptions the produced evidence fully justifies the proposed intervention.
\end{abstract}

Keywords: Potential health impact, transport traffic, air pollution, effectiveness.

\section{Actuality of the problem}

The transport traffic is known to be the major reason for air pollution in urban areas all over the modern world. Sizable proportions of emission comprise two pollutants which have direct adverse effect on health: the particles with diameter of less than 10 microns (PM10) and carbon monoxide. Among PM10 the most dangerous are particles with diameter less than 2.5 microns (PM2.5) as they could enter deep inside into lungs. The carbon monoxide links with blood hemoglobin and reduces amount of oxygen transferred to organs which poses specific threat for people with chronic cardiovascular diseases. In addition, transport traffic also emits nitrogen dioxide which adverse effects on health are not known. Finally, the majority of emission falls to carbon dioxide that plays major role in global climate change.

\section{Investigation tools for air pollution health effects}

To investigate air pollution health effects epidemiology uses laboratory studies, panel and event studies and large population studies which could further be subdivided into time-series and geographical studies. As more evidence on the health effects of air pollution is emerged the shift on intervention studies assessing mitigation of adverse health effects associated with air pollution becomes apparent. This paper discusses time-series and geographical studies which are among the main tools for the investigation of air pollution health effects.

\section{Time-series studies}

These studies investigate association between short-term, usually daily or weekly, variations in air pollution levels and health events counts, ex. number of hospitalizations or deaths, measured during the same time periods. Time-series studies 
are ecological ones as they measure exposure at group level within a well-defined area. The classical example of time-series study is the investigation of notorious smog episode in London in 1952 which showed apparent association between air pollution and daily mortality (1).

Despite ecological design confounding factors that change slowly over the time, ex. alcohol consumption, do not introduce much distortion for the association in question as we compare the same population during close and short time periods so population is used as its own control (2). For the same reason counts used instead of rates as denominator remains similar for the alike population. In contrast factors that do change over the time should be treated with caution. For example if mortality decreases as a result of booming economy and associated improved socioeconomic conditions and at the same time air pollution levels increase due to intensified industrial activity then air pollution can be spuriously interpreted as having protective role on mortality. Fortunately existing statistical methods allow filtering out such effects.

\section{Geographical studies}

These studies aimed at investigation of association between long-term exposure to air pollution and chronic health outcomes. Geographical studies compare populations that exposed to different levels of air pollution. Usually the exposure is measured at a group level and possible confounding factors at an individual level. This design also referred to as semi-ecological studies.

Geographical studies are known to be prone to confounding because they compare populations from different locations. Quite often there is a factor or number of factors which are associated with outcome and at the same time unevenly distributed between study populations which lead to distortion of association under investigation. The measurement of possible confounding factors at an individual level is aimed at control of confounding in ecological studies. In six cities study (3) one of the famous studies which used semi-ecological design the data on possible confounding factors were collected for all 8111 study participants. These data allowed strengthening the study by use of statistical approach called regression modeling for control of confounding. The six cities study found that mortality rate in the most polluted city compared to the least polluted one adjusted for the distortion effect of confounders is 1.26 times higher with corresponding $95 \%$ confidence interval (1.08 to 1.47 ).

Strengths and weaknesses of time-series and geographical studies

Time-series and geographical studies have their own advantages and limitations. Their contribution in producing evidence of air pollution effects on human health is complementary.

Time-series studies investigate short-term effects of air pollution. They are less prone to confounding by factors that change slowly over the time. However time-series studies restricted to investigation of acute effects. In addition their importance for public health is uncertain due to phenomenon called mortality displacement or harvesting (2). The exposure to air pollution episode may bring forward the death of people who already have severe health conditions. In this situation raise in mortality should follow with its decline and the found association may be attributed at large extent to mortality displacement.

Geographical studies investigate long-term air pollution effects including etiology of disease and produce evidence of clear importance. However, in these studies control for confounding becomes a challengeable issue. In addition, uncertainty exists due to the low number of locations usually participating in the study.

Potential health impact of restriction traffic in the center of Baku by $25 \%$

In 2012 the national park of cars exceeded one million units with about $60 \%$ of vehicles concentrated in the capital. Proportion of air pollution produced with cars comprises $75 \%$ from 
all man-made sources in Baku (4). The problem of traffic air pollution is being intensified with each year and becomes one of the main public health priorities in Baku.

Assuming that $50 \%$ of Baku city car traffic occurs within its center and that average annual emission for a passenger car comprises 10727 pounds (5) or $4866 \mathrm{~kg}$ the proposed intervention should approximately lead to annual decrease in emission by 364950 tons $(1 \mathrm{mln}$ cars $\times 0.6 \times 0.5 \times 0.25 \times$ $4866 \mathrm{~kg}$ ). This figure also comprises about $9.4 \%$ of annual air pollution produced by all man-made sources in Baku (0.75 x $0.5 \times 0.25 \times 100)$. However both assumptions introduce uncertainty. Firstly the center of the city is not well defined area and the percentage of cars within the center may well be above or beyond $50 \%$. Secondly average annual emission of passenger car is based on the US source; however this figure could be different for the center of Baku.

Lack of epidemiological evidence makes estimation of potential health impact of proposed intervention challengeable if even possible. However, very raw attempt can be made to estimate the degree of impact of intervention. Assuming that the results of six cities study (3) can be generalized to other cities including Baku and that center of Baku, considering its high air pollution levels, could be similar to the most polluted city of the study, mortality rate for the center of Baku compared to the least polluted city in the study should be around 1.26 times higher. Also assuming that the relationship is linear and air pollution level in the least polluted city can be neglected the decrease in traffic by $25 \%$ should decrease mortality by around

5.2\%. Also assuming that deaths evenly distributed among population and that $15 \%$ of country population leave in the center of Baku out of 53762 deaths in the country reported in 2011 (6) 8064 should have occurred within the area of proposed intervention. Finally assuming that all population in the center of Baku exposed to air pollution the total number of preventable annual deaths should be around 419 (8064 x 0.052). The counts rather rates can be used as the same population is analyzed. For the low border of $95 \% \mathrm{CI}$ the result will be more conservative -153 (8064 x 0.019). Obtained results also do not account for the fact that some chronic outcomes like lung cancer may be irreversible. Also for some health outcomes ex. respiratory diseases the true positive effect of the intervention could be delayed. Considering that the traffic noise impact on the population of Baku is $85-90 \%$ (4) and the reported number of road traffic fatalities in Azerbaijan in 2010 is 925 (7) traffic restriction in addition to reduction of air pollution will have also positive impact on these actual public health problems within the center of Baku.

The implementation of proposed traffic reduction will have difficulties mainly due to inadequate road infrastructure and vehicle-oriented mentality of the sizable part of the population. In order to be effective restriction policy should be accompanied with development of road infrastructure, improvement of public transport network and intensive construction of parking areas. Another important measure should be promotion of pedestrian movement and use of bicycles accompanied with building corresponding city capacities.

\section{Conclusion}

Despite uncertainties in assumptions used in estimation of impacts of proposed intervention the produced evidence, especially the one concerning preventable deaths, fully justifies restriction of traffic in the center of Baku by $25 \%$. The implementation of intervention however should account for obvious challenges and include promotion of corresponding behavioral change.

Financial source: Did not use financial resources.

Conflict of interest: None. 


\section{REFERENCES}

1. Ministry of Health (1954). Mortality and Morbidity During the London Fog of December 1952. London, HMSO.

2. Paul W. Environmental Epidemiology. 1st ed. Open University Press; 2006

3. Dockery DW, Pope CA et al. (1993). An association between air pollution and mortality in six U.S. cities. New England Journal of Medicine 329(24): 1753-9.

4. Piriyev Y, Gozalov S. Ecological Problems of Motor Transport of Azerbaijan. Proceedings REAL CORP 2012 Tagungsband 14-16 May 2012, Schwechat

5. Annual Emissions and Fuel Consumption for an "Average" Passenger Car. U.S. Environmental Protection Agency (1997 April). [Accessed 2013 March 21]. Available from: URL: http://msl1.mit.edu/EPA-average-ann-emit.pdf

6. Main causes of death among population. The State Statistics Committee of Azerbaijan Republic (2013). [Accessed 2013 March 21]. Available from: URL: http://www.stat.gov.az/source/healthcare/indexen.php

7. The Second Global Report on Road Safety. WHO (2013). [Accessed 2013 March 21]. Available from: URL:

http://www.who.int/violence_injury_prevention/road_ safety_status/2013/en/index.html 